\title{
Physical Consequences of Nonabelian Duality in the Standard Model
}

CHAN Hong-Mo and TSOU Sheung Tsun

January 1997 
Enquiries about copyright, reproduction and requests for additional copies of this report should be addressed to:

The Central Laboratory of the Research Councils

Library and Information Services

Rutherford Appleton Laboratory

Chilton

Didcot

Oxfordshire

OXI 1 OQX

Tel: 01235445384 Fax: 01235446403

E-mail library@rl.ac.uk

ISSN 1358-6254

Neither the Council nor the Laboratory accept any responsibility for loss or damage arising from the use of information contained in any of their reports or in any communication about their tests or investigations. 
RAL-TR-97-005

Hep-th-97-01-120

\title{
Physical Consequences of Nonabelian Duality in the Standard Model
}

\author{
CHAN Hong-Mo ${ }^{1}$ \\ Rutherford Appleton Laboratory, \\ Chilton, Didcot, Oxon OX11 OQX, U.K. \\ TSOU Sheung Tsun ${ }^{2}$ \\ Mathematical Institute, Oxford University, \\ 24-29 St. Giles', Oxford OX1 3LB, U.K.
}

\begin{abstract}
Possible physical consequences of a recently discovered nonabelian dual symmetry are explored in the standard model. It is found that both Higgs fields and fermion generations can be assigned a natural place in the dual framework, with Higgs fields appearing as frames (or " $N$-beins") in internal symmetry space, and generations appearing as spontaneously broken dual colour. Fermions then occur in exactly 3 generations and have a factorizable mass matrix which gives automatically one generation much heavier than the other two. The CKM matrix is the identity at zeroth order, but acquires mixing through higher loop corrections. Preliminary considerations are given to calculating the CKM matrix and lower generation masses. New vector and Higgs bosons are predicted.
\end{abstract}

\footnotetext{
${ }^{1}$ chanhm@ @2.rl.ac.uk

${ }^{2}$ tsou @ maths.ox.ac.uk
} 


\section{Introduction}

The long-standing interest in electric-magnetic duality $[1]-[6]$ and its nonabelian generalizations [4], [7]-[11] has seen an active revival in the last few years [12]-[16] and much effort has been devoted in finding their physical consequences.

In a previous paper [17] it was shown that Yang-Mills theories are symmetric under a generalized dual transform which reduces to the familiar Hodge star operation in the abelian case. The purpose of the present paper is to examine what physical consequences this dual symmetry might have when applied to the standard model with gauge symmetry $s u(3) \times s u(2) \times u(1)$, which seems to embody all presently known facts in particle physics apart from gravity.

We note that in contrast to many other approaches to duality adopted in the literature which aim at extending the standard model to a larger theory making use of supersymmetry and higher dimensions of space-time and/or constituents of matter (such as strings and membranes), we choose here to aim for economy. In other words, instead of looking 'beyond the standard model' as is often done, we shall remain strictly within the standard model framework in 4 space-time dimensions, and ask merely whether, within this framework, the recently discovered generalized nonabelian dual symmetry can lead to physical consequences which are as yet unknown or unexplored.

Now although this generalized dual symmetry has strictly speaking been established only for classical fields, we wish to show that when supplemented by some general known facts in quantum field theories, plus some seemingly reasonable assumptions special to our treatment, the symmetry when applied to the standard model can lead to predictions of quite considerable interest. Before studying these for the standard model in detail, however, let us first examine duality for Yang-Mills theory in general terms for indications in which areas physical consequences may be expected to arise.

We recall the generalized dual transform proposed in our earlier paper [17]:

$$
\omega^{-1}(\eta(t)) \tilde{E}_{\mu}[\eta \mid t] \omega(\eta(t))
$$




$$
=-\frac{2}{\bar{N}} \epsilon_{\mu \nu \rho \sigma} \dot{\eta}^{\nu} \int \delta \xi d s E^{\rho}[\xi \mid s] \dot{\xi}^{\sigma}(s) \dot{\xi}^{-2}(s) \delta(\xi(s)-\eta(t)),
$$

which was expressed in terms of some loop space variables $E_{\mu}[\xi \mid s]$ and its dual $\tilde{E}_{\mu}[\xi \mid s]$ describing the gauge field. The actual formalism of Yang-Mills theory in terms of these variables is unfortunately somewhat involved and delicate, but for the purpose of the present paper, we need only note the following few points. The variables $E_{\mu}[\xi \mid s]$ are nonlocal, depending on a segment of the parametrized loop $\xi$ around the point $\xi(s)$ on the loop labelled by the value $s$ of the loop parameter. The segment has width $\epsilon$ which eventually is to be taken to zero, and in the limit $\epsilon \rightarrow 0$,

$$
E_{\mu}[\xi \mid s] \rightarrow F_{\mu \nu}(\xi(s)) \dot{\xi}^{\nu}(s)
$$

where the dot denotes differentiation with respect to the loop parameter $s$. In other words, in the limit of zero segmental width, $E_{\mu}[\xi \mid s]$ is just the YangMills field at the point $\xi(s)$ dotted into the tangent to the loop at that point. However, the rules of operation are such that the limit $\epsilon \rightarrow 0$ is to be taken only after all loop differentiations and integrations, such as that occurring in the dual transform (1.1), have already been performed. This generalized dual transform is thus a rather complicated affair, but is known to reduce just to the Hodge star for the abelian theory and in the general case to share the property with the Hodge star of being its own inverse apart from a sign. A new feature, however, which did not occur in the abelian theory, is the matrix $\omega(x)$ which transforms from the internal symmetry frame ( $U$-frame) in which fields of the direct formulation are measured to the frame ( $\tilde{U}$-frame) in which fields of the dual formulation are measured. As we shall see, this quantity will acquire a major significance in our future discussion.

The result of our earlier paper was that Yang-Mills theory is symmetric under the generalized transform (1.1), and our present purpose is to explore the consequences. We note first that this symmetry implies that in addition to the original gauge invariance, say $G$, the theory will possess a further gauge invariance (the dual invariance) $\tilde{G}$, having the same group strucutre but an opposite parity to the first, so that it has in all a $G \times \tilde{G}$ local gauge invariance. Further, it implies that a dual potential $\tilde{A}_{\mu}(x)$ exists which plays 
a role exactly dual to that of the ordinary Yang-Mills potential $A_{\mu}(x)$. Notice that $\tilde{A}_{\mu}(x)$ does not represent an additional degree of freedom to $A_{\mu}(x)$ since $\tilde{A}_{\mu}(x)$ is related to the dual field variable $\tilde{E}_{\mu}[\xi \mid s]$ in exactly the same way as $A_{\mu}(x)$ is related to $E_{\mu}[\xi \mid s]$, and $\tilde{E}_{\mu}$ is given in terms of $E_{\mu}$ via the generalized transform (1.1). However, $\tilde{A}_{\mu}(x)$ provides an alternative description of the gauge field to that provided by $A_{\mu}(x)$, and for certain phenomena, the former may be much more convenient than the latter. For example, in terms of $\tilde{A}_{\mu}(x)$ the phase transport of the wave function of a (colour) magnetic charge is simple, being just exp $i \tilde{g} \tilde{A}_{\mu}(x) d x^{\mu}$ from $x$ to a neighbouring point $x+$ $d x^{\mu}$, whereas an expression of the same quantity in terms of $A_{\mu}(x)$, though presumably possible, would be extremely complicated. In particular, the Wilson operator:

$$
A(C)=\operatorname{Tr}\left(P \exp i g \oint_{C} A_{\mu} d x^{\mu}\right)
$$

in the words of 't Hooft[10], measures magnetic flux through $C$ and creates electric flux along $C$. Then by dual symmetry the operator:

$$
B(C)=\operatorname{Tr}\left(P \exp i \tilde{g} \oint_{C} \tilde{A}_{\mu} d x^{\mu}\right)
$$

should measure electric flux through $C$ and create magnetic flux along $C$. And indeed, using the generalized dual transform (1.1), one can show[18] that this operator $B(C)$ does satisfy the following commutation relation with $A(C)$, which was used by t' Hooft to abstractly define the $B(C)$ operator:

$$
A(C) B\left(C^{\prime}\right)=B\left(C^{\prime}\right) A(C) \exp 2 \pi i n / N,
$$

where $n$ is the number of times $C^{\prime}$ winds around $C$ and $N$ is for the gauge group $S U(N)$.

Using the commutation (1.5), 't Hooft derived the important result that if the electric field is confined, then the magnetic field is in the Higgs phase, and vice versa. Suppose now that $A(C)$ is confined, then $B(C)$ should be in the Higgs phase, and its corresponding potential $\tilde{A}_{\mu}$ representing the dual gauge boson should then acquire a mass and be permitted to propagate freely through space. At first sight, this may seem contradictory to the statement that $A_{\mu}$ (colour) is confined, meaning that $A_{\mu}$ can be nonvanishing only inside 
hadrons, since $A_{\mu}$ and $\tilde{A}_{\mu}$ are supposed to represent just the same degrees of freedom. We believe, however, that this is not the case. By confinement we mean that coloured objects cannot propagate freely in space, and a gluon $A_{\mu}$, being coloured, has therefore to remain inside a hadron. The dual gluon $\tilde{A}_{\mu}$, however, is not coloured (electrically, that is). This can be seen in the generalized dual transform of (1.1). Under an ordinary colour gauge (that is, in our present language, an electric $U$-gauge) transformation $S(x)$ :

$$
E_{\mu}[\xi \mid s] \rightarrow S(\xi(s)) E_{\mu}[\xi \mid s] S^{-1}(\xi(s)) .
$$

This change, however, is compensated in (1.1) by a corresponding transformation in the matrix $\omega(x)$, which transforms under $S(x)$ as:

$$
\omega(x) \rightarrow \omega(x) S^{-1}(x),
$$

leaving thus $\tilde{E}_{\mu}[\eta \mid t]$, and hence also $\tilde{A}_{\mu}$, invariant. Like $\tilde{E}_{\mu}[\eta \mid t], \tilde{A}_{\mu}$ is magnetically coloured but electrically colourless. It has thus no reason to be confined. And although $A_{\mu}$ and $\tilde{A}_{\mu}$ represent the same degrees of freedom, specifying an $\tilde{A}_{\mu}$ outside hadrons in free space is not double-counting since there $A_{\mu}$, by virtue of confinement, does not propagate. In other words, we are saying that although the gluon, being coloured, is confined inside hadrons, the degree of freedom it represents can still manifest itself in the free space outside hadrons as a massive, colour-magnetically charged, but colour-electrically neutral, dual gluon.

Perhaps a more physical way of presenting the above conclusion, which may make it easier to visualize, is to picture $\omega(x)$ itself as a field. It represents then a colour dyon, carrying both a colour electric and a colour magnetic charge, transforming under $\tilde{U}$-transformations $\tilde{S}(x)$ as the fundamental representation and under $U$-transformations $S(x)$ as the conjugate fundamental representation, thus:

$$
\omega(x) \longrightarrow \tilde{S}(x) \omega(x) S^{-1}(x) .
$$

The dual field $\tilde{E}_{\mu}$ in (1.1) can then be pictured as a composite object (a bound state!) formed from an (electrically) coloured field $E_{\mu}$ belonging to 
the adjoint representation and an $\omega-\bar{\omega}$ pair, in such a way as to make the whole colour-electrically neutral, though colour-magnetically charged. The result is thus, in a sense, also a hadron, and has the right to propagate through space as any other hadron. That being the case, there seems no reason why they cannot be detected experimentally in principle.

The observation in the preceding paragraph about the matrix $\omega(x)$ brings us to another point in duality which may have observable consequences. Although introduced at first by us in all innocence as just a transformation matrix to keep track of the gauge invariance, this $\omega(x)$ is seen to have gradually acquired more and more physical attributes. Thus, for example, it was seen already in earlier papers $[16,17]$ that in the presence of charges, whether electric or magnetic, $\omega(x)$ will have to be patched. This means that it cannot arbitrarily be put to unity everywhere by a gauge transformation as one might expect for a mere transformation matrix. Now, we find further that $\omega(x)$ can be combined with other fields to fundamentally change their physical behaviour. We propose therefore to consider promoting $\omega(x)$ to the status of a genuine field variable. Now in the classical field theory, $\omega(x)$ is a unitary matrix, being an element of the gauge group. By promoting it to a physical field, we mean, presumably, allowing it to fluctuate about its classical (vacuum) value. We ask in such a case what physical significance it might have.

We note first that being a transformation matrix in internal symmetry space, $\omega(x)$ is invariant under Lorentz transformations. It takes a wave function for an electric charge to one for a magnetic charge, and if we give opposite parities to the two wave functions, as would seem natural, the matrix $\omega(x)$ would be a space-time pseudoscalar. Under a $U$-gauge (electric) transformation, the rows of $\omega(x)$ transform as the conjugate fundamental representation, while under a $\tilde{U}$-gauge (magnetic) transformation, its columns transform as the fundamental representation. Further, its vacuum value being a unitary matrix, its rows and columns all have vacuum values of unit length. In particular, then, for an $S U(2)$ theory, a row of $\omega(x)$ would represent a space-time pseudoscalar, isodoublet field with a vacuum value of fixed (unit) length, as is wanted for the Higgs field of the electroweak theory. It is thus interesting 
to entertain the possibility that the rows and columns of $\omega(x)$ are indeed the Higgs fields in the theory responsible for symmetry breaking. If this turns out to be so, we would find ourselves in the happy position where the Higgs fields required in the theory, which normally we have to introduce by hand to give the desired symmetry breaking pattern, actually arise in a natural manner as just the transformation matrix between the direct and dual gauge frames of the theory. It may even mean that certain aspects in the symmetry breaking pattern of the theory can be predicted.

The classical considerations of our previous papers, however, give only the vacuum configuration of the 'Higgs fields' as $\omega(x)$ but leave open the question of how exactly the 'promotion' of $\omega(x)$ to physical Higgs fields $\phi(x)$ is to be effected. Our proposal for doing so will be given below in section 3 when applying the idea to the standard model. We note that the vacuum expectation values $\omega(x)$ themselves have to do only with the pattern of symmetry breaking but not with the symmetry breaking scales. These latter are governed by how easily the Higgs fields can fluctuate from their vacuum values, and by how rapidly these fluctuations are allowed to vary from point to point in space-time. These pieces of information are encoded in standard formulations in the sizes of the kinetic energy term $\partial_{\mu} \phi \partial^{\mu} \phi$, the 'mass' term $-\mu^{2} \phi^{2}$ and in the quartic term $\lambda \phi^{4}$ of the Higgs action, relative both to one another and to the rest of the action. These parameters are ultimately related to the masses of the Higgs bosons and the Higgsed gauge bosons, which are thus still free parameters in the present theory to be determined by phenomenology. Later on, we shall mention some possibilities whereby duality may also help in constraining these parameters.

Supposing that Higgs fields can indeed be constructed in this way, then the $\tilde{E}_{\mu}$ field which was pictured as a composite formed from combining the gauge field $E_{\mu}$ with an $\omega-\bar{\omega}$ pair can be considered as a genuine bound state of the gauge and Higgs fields. Now it has already been noted by 't Hooft [10] that a confined system with scalar fields in the fundamental representation of the gauge group can appear very similar to a system in the Higgs phase, since the fundamental 'Higgses' can combine with coloured fields to form colourless bound states which need no longer be confined. Our picture here 
can thus be regarded as just a special case of the 't Hooft scenario, in which the naturally occurring fundamental scalar field $\omega(x)$ plays the role of the Higgs field, and combines with the confined gluon to give the massive, freely propagating dual gluon. What is slightly unusual is that both pictures here apply concurrently.

We have considered above only the pure gauge theory. When charges are introduced, then further consequences of duality may result. It has been shown that charges in one description appear as monopoles in the dual description, and monopoles, being topological obstructions, can only have certain charges prescribed by the topology of the gauge group. Thus, given the electric charges of a theory, one can deduce what magnetic charges can occur. Further, 't Hooft's result quoted above implies that if the electric group is unbroken and confined, then the dual group is broken and Higgsed, and vice versa. Hence given the charges we know, we have a fair idea how their dual charges will behave. It would therefore be interesting to enquire whether any of these dual charges may correspond to quantum numbers already known to us but yet unexplained. We have in mind in particular the question of whether the generation index which is so far entirely phenomenological, with no theoretical indication of its origin, can be interpreted as dual colour. This last has the advantage of occurring naturally in the gauge theory and of numbering exactly 3 , as seems indicated for the generation index by recent experiment. Such questions, however, are best discussed below where we examine in our framework the standard model in detail.

\section{Monopoles of the Standard Model}

We begin by collecting together some bits of information on the standard model essential to our discussion later which though published already in the literature [19] may yet not be too widely known.

In most (perturbative) applications of gauge theories, one needs to specify only the gauge Lie algebra, but for studying monopoles, one needs also the gauge group. Different groups may correspond to the same algebra. For example, both the groups $S U(2)$ and $S O(3)=S U(2) / \mathbf{Z}_{2}$ correspond to the 
same algebra $s u(2),{ }^{3}$ and whether monopoles charges may exist in a theory depends on whether the gauge group is $S U(2)$ or $S O(3)$.

Given the gauge Lie algebra, the gauge group of a theory is to be determined by examining what fields occur in the theory. [20,21] For example, the maximal group generated by $s u(2)$ is $S U(2)$, but in the pure Yang-Mills theory where only the gauge fields in the adjoint representation occur, 2 elements in $S U(2)$ differing by a sign will have the same physical effect and have thus to be identified. Hence the gauge group of the theory is $S U(2) / \mathbf{Z}_{2}=S O(3)$ and not $S U(2)$ itself.

An analysis along these lines taking account of all presently known particles and fields gives as the gauge group of the standard model not the maximal group $S U(3) \times S U(2) \times U(1)$ generated by the algebra $s u(3) \times s u(2) \times u(1)$, but a group obtained by identifying the following sextets of elements in the maximal group:

$$
(c, f, y),\left(c c_{1}, f, y y_{1}\right),\left(c c_{2}, f, y y_{2}\right),\left(c, f f_{-}, y y_{-}\right),\left(c c_{1}, f f_{-}, y y_{-} y_{1}\right),\left(c c_{2}, f f_{-}, y y_{-} y_{2}\right) \text {, }
$$

where $c, f$ and $y$ are elements respectively of $S U(3), S U(2)$ and $U(1)$, with:

$$
\begin{aligned}
c_{r} & =\exp \frac{2 \pi i r}{\sqrt{3}} \lambda_{8}, r=1,2 \\
f_{-} & =\exp 2 \pi i T_{3} \\
y_{r} & =\exp 4 \pi i r Y, r=1,2 \\
y_{-} & =\exp 6 \pi i Y
\end{aligned}
$$

We shall call this group $U_{2,3}$, a version of $S(U(3) \times U(2))$. We note also that when restricted only to the electroweak sector, the gauge group is $U(2)=S U(2) \times U(1) / \mathbf{Z}_{2}$, and when restricted only to chromodynamics and electromagnetism, the gauge group is $U(3)=S U(3) \times U(1) / \mathbf{Z}_{3}$; in neither case is the gauge group the maximal group generated by the corresponding gauge Lie algebra.

\footnotetext{
${ }^{3}$ We shall use capitals for groups but small letters for algebras. Although it is more correct to denote semi-simple algebras as direct sums, we shall adhere to the product notation to avoid confusion.
} 
The topology of the gauge group determines the values that the monopole charges of the theory can take. Thus, generalizing the arguments leading to the Dirac quantization condition for monopole charges in electromagnetism, one can deduce in general that monopole charges are given by the elements of the fundamental group $\pi_{1}(G)$ of the gauge group $G$. These are the homotopy classes of closed curves in $G$ where members of each class are curves continuously deformable within $G$ into one another. In particular, for a $U(1)$ theory when the gauge group has the topology of the circle, $\pi_{1}(G)=\mathbf{Z}$; it follows then that monopole charges here are labelled by integers, namely the winding numbers around the circle representing $U(1)$, which is the old Dirac result.

Applied to the gauge group $U_{2,3}$, this implies that monopole charges of the standard model are also labelled by integers, where a monopole labelled by $n$ can be regarded as carrying simultaneously: ${ }^{4}$

(a) a dual colour charge $\zeta=\exp 2 \pi i n / 3$;

(b) a dual weak isospin charge $\eta=(-1)^{n}$;

(c) a dual weak hypercharge $\tilde{Y}=2 \pi n / 3 g_{1}$.

Any monopole in the theory will have to carry the combination of charges listed in (2.3) for some choice of integer $n$. We note that in (2.3) dual colour and dual weak isospin take values only in $\mathbf{Z}_{3}$ and $\mathbf{Z}_{2}$ respectively. Thus for dual weak isospin, $\eta=+$ corresponds to the vacuum, $\eta=-$ to a monopole, and a monopole is its own conjugate; but for dual colour, $\zeta=1$ corresponds to the vacuum, while $\zeta=\exp 2 \pi i / 3$ and $\zeta=\exp 4 \pi i / 3$ correspond to monopoles of conjugate charges.

So far, one has made no use yet of dual symmetry. For the standard model, dual symmetry implies that in addition to the original gauge symmetry generated by the algebra $s u(3) \times s u(2) \times u(1)$, there is a further gauge symmetry generated by another algebra $\widetilde{s u}(3) \times \widetilde{s u}(2) \times \tilde{u}(1)$ with the same structure but opposite parity. Moreover, dual symmetry says that

\footnotetext{
${ }^{4}$ We use here a different normalization convention for the gauge fields and their couplings from that used in our earlier publications, e.g. [19], so as to conform with the usual practice in the literature on the standard model.
} 
charges in one gauge symmetry are monopoles in the dual gauge symmetry and vice versa. Hence, the monopole charges of $U_{2,3}$ listed in (2.3) above can also be regarded as ordinary (electric) charges of the dual symmetry $\widetilde{s u}(3) \times \widetilde{s u}(2) \times \tilde{u}(1)$. But charges of gauge symmetries are usually assigned to representations of the gauge symmetry. So we have to ask to what representations of the dual symmetries the monopoles in (2.3) should correspond. The answer is as follows:

$$
\begin{aligned}
\text { (a) } \zeta & =1 \sim \text { dual colour singlet } \tilde{\mathbf{1}} \\
\zeta & =\exp 2 \pi i / 3 \sim \text { dual colour triplet } \tilde{\mathbf{3}} \\
\zeta & =-\exp 4 \pi i / 3 \sim \text { dual colour antitriplet } \overline{\tilde{\mathbf{3}}} \\
\text { (b) } \eta & =+\sim \text { dual weak isospin singlet } \tilde{\mathbf{1}}, \\
\eta & =-\sim \text { dual weak isospin doublet } \tilde{\mathbf{2}}, \\
\text { (c) } \tilde{Y} & =n \tilde{g}_{1} / 3 .
\end{aligned}
$$

Besides representations of the dual symmetries, the monopoles in (2.3), when considered as charges in these symmetries, have to be further characterized by their coupling strengths, $\tilde{g}_{3}, \tilde{g}_{2}$ and $\tilde{g}_{1}$, to the dual gauge fields $\tilde{C}_{\mu}(x), \tilde{W}_{\mu}(x)$ and $\tilde{B}_{\mu}(x)$ for respectively dual colour, dual weak isospin, and dual weak hypercharge. Furthermore, these couplings themselves ought to be related to the usual colour, weak isospin, and weak hypercharge couplings $g_{3}, g_{2}$ and $g_{1}$ by conditions similar to the familiar Dirac condition relating the strengths of quantized electric and magnetic charges. The exact form of these generalized Dirac conditions depend on how the various quantities are normalized. For weak hypercharge, the condition is the same as for electromagnetism, namely:

$$
g_{1} \tilde{g}_{1}=2 \pi,
$$

as already implied in (2.3) and (2.4). For colour and weak isospin, if we follow the standard convention and write the free action as:

$$
\mathcal{A}_{0}=\frac{1}{4} \int d^{4} x \operatorname{Tr}\left(F_{\mu \nu} F^{\mu \nu}\right)+\int d^{4} x \bar{\psi}\left(i \partial_{\mu} \gamma^{\mu}-m\right) \psi
$$

with:

$$
F_{\mu \nu}=\partial_{\nu} C_{\mu}-\partial_{\mu} C_{\nu}+i g_{3}\left[C_{\mu}, C_{\nu}\right]
$$




$$
C_{\mu}=C_{\mu}^{\alpha} \lambda_{\alpha} / 2, \alpha=1, \ldots, 8
$$

for colour, and

$$
\begin{gathered}
F_{\mu \nu}=\partial_{\nu} W_{\mu}-\partial_{\mu} W_{\nu}+i g_{2}\left[W_{\mu}, W_{\nu}\right], \\
W_{\mu}=W_{\mu}^{\rho} \tau_{\rho} / 2, \rho=1,2,3,
\end{gathered}
$$

$\lambda_{\alpha}$ and $\tau_{\rho}$ being respectively the Gell-Mann and Pauli matrices, and similar formulae also for the dual quantities, then the generalized Dirac conditions read as follows:[18]

$$
g \tilde{g}=4 \pi
$$

for both colour and weak isospin. With these conditions the translation into the dual description of the information in (2.3) on the monopole charges is now complete.

Conversely, charges in the original (direct) symmetry $s u(3) \times s u(2) \times u(1)$ can also be considered as monopoles of the dual symmetry. However, not knowing the experimental spectrum of the dual charges, if any exist, we cannot as yet specify the dual gauge group, nor yet the admissible charges its monopoles can have. However, if we assume that the dual gauge group is another $U_{2,3}$, say $\tilde{U}_{2,3}$, so that its monopole charges are again given by (2.3), then it is seen that all known particles can be accommodated as monopoles of the dual group with appropriate choices of $\tilde{n}$. One can thus assume without any inconsistency that the overall gauge group of the standard model is $U_{2,3} \times \tilde{U}_{2,3}$, although our considerations in what follows will not depend on this assumption.

\section{The Promotion of $\omega$ to Higgs Fields}

We wish now to specify what we mean by promoting the rows and columns of the transformation matrix $\omega(x)$ to be Higgs fields.

We recall that $\omega(x)$ was originally conceived as the matrix relating the internal symmetry $U$-frame to the dual symmetry $\tilde{U}$-frame. The rows of $\omega$ therefore transform as the conjugate fundamental representation of the $U$ symmetry, i.e. as $\overline{\mathbf{3}}$ of colour or $\overline{\mathbf{2}}$ of weak isospin, while its columns transform as the fundamental representation of the dual $\tilde{U}$-symmetry, i.e. as $\mathbf{3}$ of dual 
colour or 2 of dual weak isospin. Let us then introduce Higgs field $\phi^{(i)}$ and $\tilde{\phi}^{(i)}$, with the index (i) running over $1,2,3$ for $S U(3)$ and over 1,2 for $S U(2)$, having the above transformation properties under respectively the $U$ and $\tilde{U}$ symmetries.

We want the vacuum expectation values of these Higgs fields to be such as to give an orthonormal triad for $S U(3)$ and an orthonormal dyad for $S U(2)$, thus:

$$
\begin{aligned}
& \phi_{j}^{(i)}(x) \longrightarrow \phi_{0}^{(i)} v_{j}^{(i)}(x), \\
& \tilde{\phi}_{j}^{(i)}(x) \longrightarrow \tilde{\phi}_{0}^{(i)} \tilde{v}_{j}^{(i)}(x),
\end{aligned}
$$

where at any $x$ :

$$
\begin{aligned}
& \sum_{k} \bar{v}_{k}^{(i)} v_{(j)}^{k}=\bar{v}^{(i)} \cdot v_{(j)}=\delta_{(j)}^{(i)}, \\
& \sum_{k} \overline{\tilde{v}}_{k}^{(i)} \tilde{v}_{(j)}^{k}=\overline{\tilde{v}}^{(i)} \cdot \tilde{v}_{(j)}=\delta_{(j)}^{(i)},
\end{aligned}
$$

so that we have for the transformation matrix $\omega(x)$ at any $x$ :

$$
\omega_{j}^{k}=\sum_{(i)} v_{j}^{(i)} \overline{\tilde{v}}_{(i)}^{k} .
$$

We notice that the quantities $v_{j}^{(i)}$ and $\tilde{v}_{j}^{(i)}$ are actually just the frame vectors in respectively the direct and dual description of internal symmetry space. In promoting them to dynamical variables as the Higgs fields $\phi_{j}^{(i)}$ and $\tilde{\phi}_{j}^{(i)}$ as we do here is thus similar in spirit to the Palatini treatment of gravity in terms of the frame vectors or vierbeins as dynamical variables, [22] with the transformation matrix $\omega$ here playing the role of the metric.

Next, if we follow the standard procedure for Higgs fields, we would wish presumably to obtain their vacuum expectations $v_{j}^{(i)}$ and $\tilde{v}_{j}^{(i)}$ as usual by minimizing some potential $\mathcal{V}[\phi, \tilde{\phi}]$. Let us see what sort of a potential we need. First, of course, $\mathcal{V}$ should be invariant under the $U$ and $\tilde{U}$-transformations given the above transformation properties of $\phi$ and $\tilde{\phi}$. Second, we want $\mathcal{V}$ to be symmetric under permutations of the index $(i)$ of $\phi^{(i)}$ and $\tilde{\phi}^{(i)}$, given that the $\phi^{(i)}$ 's for different $(i)$ have exactly equivalent status. Third, given ' $t$ Hooft's result that if one phase is Higgsed then the dual phase is confined, 
we want $\mathcal{V}$ to be such that if $\phi_{0}>0$, then $\tilde{\phi}_{0}=0$, and vice versa. Fourth, given that the resulting theory should be renormalizable, we want $\mathcal{V}$ to be a polynomial in $\phi$ and $\tilde{\phi}$ of degree no higher than 4 . Notice that although the potential is required to be symmetric under permutations of $\phi^{(i)}$, one expects in general that this permutation symmetry will also be spontaneously broken along with the continuous symmetry giving then different values to $\phi_{0}^{(i)}$ for different $(i)$. Can we find an appropriate potential with such properties and yet have the above vacuum configuration as its minima?

We suggest the following:

$$
\mathcal{V}[\phi, \tilde{\phi}]=V[\phi]+V[\tilde{\phi}]
$$

where:

$$
V[\phi]=-\mu \sum_{(i)}\left|\phi^{(i)}\right|^{2}+\lambda\left\{\sum_{(i)}\left|\phi^{(i)}\right|^{2}\right\}^{2}+\kappa \sum_{(i) \neq(j)}\left|\bar{\phi}^{(i)} \cdot \phi^{(j)}\right|^{2},
$$

with the stipulation that $\mu$ is odd while $\lambda$ and $\kappa$ are even under the dual transform, namely $\tilde{\mu}=-\mu, \tilde{\lambda}=\lambda>0, \tilde{\kappa}=\kappa>0$. This potential is interesting in that its minimum occurs (for $\mu>0$ ) when the $\phi$ 's are mutually orthogonal and when $\sum_{(i)}\left|\phi^{(i)}\right|^{2}=\mu / 2 \lambda$, independently of the individual lengths of the different $\phi$ 's. The minimum has thus a symmetry greater than that contained in the potential which is only symmetric under permutations of the $\phi^{(i)}$ 's, so that different vacua from that degenerate set contained in the minimum can be physically inequivalent. A vacuum chosen randomly from the set will in general have different vacuum expectations values for all $\phi^{(i)}$ and we shall develop our future arguments for this general case for which the potential (3.7) applies. Our considerations below, however, will not depend on the explicit form of the potential.

With the above proposal as Higgs fields, let us examine the familiar case of electroweak symmetry breaking. Here, of course, we know from experiment exactly how the symmetry should be broken, namely as in the SalamWeinberg manner, so that a rederivation of this result will serve as a check on the validity of the present approach. We note first that in contrast to most theories, one is not allowed here to choose whatever Higgs fields one 
wants to give the desired symmetry breaking pattern but is obliged to introduce 2 (and only 2) weak isodoublets $\phi^{(1)}$ and $\phi^{(2)}$ as our Higgs fields. The weak hypercharges of $\phi^{(i)}$, however, which have so far not entered into our argument with $\omega$, are not yet completely specified. Given that our present electroweak theory has gauge group $U(2)$, as explained in the last section, it follows that $\phi^{(1)}$ and $\phi^{(2)}$ can only have weak hypercharges $n / 2$ with $n$ being an odd integer (positive or negative). However, we are still free to choose $\phi^{(1)}$ and $\phi^{(2)}$ having various odd half-integral values, and the resultant pattern of symmetry breaking will depend on the choice.

An easy way to deduce the symmetry breaking pattern for some given choice of hypercharges for the Higgs fields is to examine the mass matrix for the gauge bosons arising from the kinetic energy term in the action for the Higgs fields:

$$
\sum_{(i)} D_{\mu} \phi^{(i)} D^{\mu} \phi^{(i)}
$$

where we have insisted, as in the Higgs potential, that symmetry should be maintained between the 2 Higgs fields $\phi^{(1)}$ and $\phi^{(2)}$. Both these Higgs field having been designated as weak isospin doublets, it follows that the covariant derivative is:

$$
D_{\mu}=\partial_{\mu}-i g_{2} W_{\mu}^{\alpha}(x) \frac{\tau_{\alpha}}{2}-i g_{1} \frac{n}{2} B_{\mu}(x)
$$

for both, each with its appropriate choice of $n$ for weak hypercharge. The mass matrix for the gauge bosons is then given as:

$$
W_{\mu}^{\alpha^{\prime}} M_{\alpha^{\prime} \beta^{\prime}} W^{\beta^{\prime} \mu}=\sum_{(i)} \bar{\phi}_{V}^{(i)}\left\{-g_{2} W_{\mu}^{\alpha} \frac{\tau_{\alpha}}{2}-g_{1} \frac{n}{2} B_{\mu}\right\}^{2} \phi_{V}^{(i)}
$$

where the primed indices $\alpha^{\prime}, \beta^{\prime}$ on the left-hand side are meant to run over $1,2,3$ and 0 , with $W_{\mu}^{0}=B_{\mu}$, while the vacuum expection values $\phi_{V}^{(1)}$ and $\phi_{V}^{(2)}$ of the Higgs fields on the right may be taken as:

$$
\phi_{V}^{(1)}=\left(\begin{array}{c}
v \\
0
\end{array}\right), \phi_{V}^{(2)}=\left(\begin{array}{c}
0 \\
w
\end{array}\right)
$$

in conformity with (3.1) and (3.3) above. 
From (3.10), it is clear that $M_{\alpha^{\prime} \beta^{\prime}}$ is diagonal for $\alpha^{\prime}$ or $\beta^{\prime}=1,2$, namely that

$$
M_{\alpha^{\prime} \beta^{\prime}}=\frac{g_{2}^{2}}{4}\left(v^{2}+w^{2}\right) \delta_{\alpha^{\prime} \beta^{\prime}}
$$

which means that the gauge bosons $W_{\mu}^{1,2}$ are unmixed, with each acquiring a mass of $\left(g_{2} / 2\right) \sqrt{v^{2}+w^{2}}$. The other 2 gauge bosons, $\alpha^{\prime}, \beta^{\prime}=3,0$, on the other hand, will have the following mass sub-matrix:

$$
M_{\alpha^{\prime} \beta^{\prime}}=\frac{1}{4}\left(\begin{array}{cc}
g_{2}^{2}\left(v^{2}+w^{2}\right) & g_{2} g_{1}\left(n_{(1)} v^{2}-n_{(2)} w^{2}\right) \\
g_{2} g_{1}\left(n_{(1)} v^{2}-n_{(2)} w^{2}\right) & g_{1}^{2}\left(n_{(1)}^{2} v^{2}+n_{(2)}^{2} w^{2}\right)
\end{array}\right)
$$

for which mixing will in general occur (except when $n_{(1)}=n_{(2)}$ and $v=w$ ). Furthermore, both the eigenstates will in general acquire a mass, in which case the electroweak $U(2)$ symmetry will be completely broken. The only situation when this will not happen is when $n_{(1)}=-n_{(2)}=n$, namely when the 2 Higgs field $\phi^{(i)}$ have opposite hypercharges, in which case the matrix becomes:

$$
M_{\alpha^{\prime} \beta^{\prime}}=\frac{1}{4}\left(v^{2}+w^{2}\right)\left(\begin{array}{cc}
g_{2}^{2} & n g_{2} g_{1} \\
n g_{2} g_{1} & n^{2} g_{1}^{2}
\end{array}\right)
$$

which is of rank 1 , and hence has one vanishing eigenvalue. As a result, the electroweak symmetry $U(2)$ will be broken down to a residual $U(1)$ with the zero-mass eigenstate as the photon.

One sees therefore that the standard Salam-Weinberg theory does occur as a special case of the Higgs scheme proposed above, corresponding to the choice $n=1$ and $v=w$. Different choices of $n$ give different hypercharges to the Higgs fields, and the simplest choice $n=1$ is the one needed to give Yukawa couplings to the existing quarks and leptons. Different choices for $v \neq w$ on the other hand will change only the predictions for the Higgs bosons themselves which are not yet discovered. Thus, as far as those of its essential features are concerned which have so far been been tested by experiment, the Salam-Weinberg theory is the unique solution in the suggested approach, so long as it is stipulated that a residual symmetry remains after the symmetry is broken. It seems therefore that the idea of promoting the transformation matrix $\omega$ to Higgs fields is quite viable, having passed the test in the only 
example of spontaneous symmetry breaking in particle physics which has so far been confirmed by experiment.

The choice of $v=w$ would be natural if one assumes as in the minimal single Higgs model that $\phi^{(2)}$ is the C-conjugate of $\phi^{(1)}$. Here, however, we have no good theoretical reason to make this special choice. Although there is also nothing against doing so, it would seem perhaps more natural in view of the proposed interpretation of Higgs fields as frame vectors to regard $\phi^{(1)}$ and $\phi^{(2)}$ as independent fields. In that case, there will be more Higgs bosons, but also naturally different values for $v$ and $w$, with the advantage of giving different masses to $u$ - and $d$-type quarks without requiring widely different values for their Yukawa couplings.

\section{The Breaking of Dual Colour}

From dual symmetry, one deduces that dual to the usual su(3) clour symmetry, there is an $\widetilde{s u}(3)$ symmetry for dual colour, and from 't Hooft's argument [10] plus the empirical fact that colour is unbroken and confined, one deduces that this $\widetilde{s u}(3)$ for dual colour will be broken and Higgsed.

How is the breaking of $\widetilde{s u}(3)$ to be achieved? At this point, we enter an uncharted domain with no longer experimental facts or previous experience to guide us, but according to the suggestions above, the symmetry breaking is to be attained by introducing as Higgs fields 3 triplets $\tilde{\phi}^{(a)},(a)=1,2,3$ of dual colour corresponding to the rows of the transformation matrix $\omega$. These $\tilde{\phi}$ 's can carry also dual or magnetic hypercharges which, according to the analysis in Section 2 , can only take the values $(\tilde{n}+1 / 3) \tilde{g}_{1}$, with $\tilde{n}$ an integer (positive or negative), and $\tilde{g}_{1}=2 \pi / g_{1}$. As with the breaking of the electroweak symmetry treated in the preceding section, the symmetry breaking pattern here will depend on what dual hypercharges are assigned to the Higgs fields $\tilde{\phi}^{(a)}$.

To identify the breaking pattern, let us examine again the gauge boson mass matrix arising from the kinetic energy of the Higgs fields:

$$
\sum_{(a)} D_{\mu} \tilde{\tilde{\phi}}^{(a)} D^{\mu} \tilde{\phi}^{(a)},
$$


with

$$
D_{\mu}=\partial_{\mu}-i \tilde{g}_{3} \tilde{C}_{\mu}^{\alpha} \frac{\lambda_{\alpha}}{2}-i \tilde{g}_{1}\left(\tilde{n}_{(a)}+1 / 3\right) \tilde{B}_{\mu},
$$

where $\tilde{C}_{\mu}$ and $\tilde{B}_{\mu}$ are respectively the gauge potentials for dual colour and dual hypercharge which, through our previous work[17], we know exist. The mass matrix for the gauge bosons is given as:

$$
\tilde{C}_{\mu}^{\alpha^{\prime}} M_{\alpha^{\prime} \beta^{\prime}} \tilde{C}^{\alpha^{\prime} \mu}=\sum_{(a)} \overline{\tilde{\phi}}_{V}^{(a)}\left[-\tilde{g}_{3} \tilde{C}_{\mu}^{\alpha} \frac{\lambda_{\alpha}}{2}-\tilde{g}_{1}(\tilde{n}+1 / 3) \tilde{B}_{\mu}\right]^{2} \tilde{\phi}_{V}^{(a)}
$$

where $\alpha^{\prime}, \beta^{\prime}=0,1, \ldots, 8$ with $\tilde{C}_{\mu}^{0}=\tilde{B}_{\mu}$, and $\tilde{\phi}_{V}^{(a)}$, the vacuum expectations for the Higgs fields, can be chosen as:

$$
\tilde{\phi}_{V}^{(1)}=\left(\begin{array}{l}
x \\
0 \\
0
\end{array}\right), \tilde{\phi}_{V}^{(2)}=\left(\begin{array}{l}
0 \\
y \\
0
\end{array}\right), \tilde{\phi}_{V}^{(3)}=\left(\begin{array}{l}
0 \\
0 \\
z
\end{array}\right) .
$$

A similar analysis to that given for electroweak symmetry breaking in Section 3 then shows that the dual gluons $\tilde{C}_{\mu}^{\alpha}$ for $\alpha=1,2,4,5,6,7$ remain unmixed but acquire respectively the following masses:

$$
\begin{aligned}
\alpha=1,2: M_{\alpha} & =\frac{\tilde{g}_{3}}{2} \sqrt{x^{2}+y^{2}} \\
\alpha=4,5: M_{\alpha} & =\frac{\tilde{g}_{3}}{2} \sqrt{x^{2}+z^{2}} \\
\alpha=7,6: M_{\alpha} & =\frac{\tilde{g}_{3}}{2} \sqrt{y^{2}+z^{2}}
\end{aligned}
$$

whereas the other 2 components $\tilde{C}_{\mu}^{\alpha}$ for $\alpha=3,8$ will in general mix with each other and with the dual hypercharge potential $\tilde{B}_{\mu}$. As for the breaking of the electroweak symmetry studied in Section 3 , the mass matrix here will leave a residual symmetry only for exceptional choices of the integers $\tilde{n}_{(1)}, \tilde{n}_{(2)}, \tilde{n}_{(3)}$ and of the parameters $x, y, z$. For example, for $\tilde{n}_{(1)}=\tilde{n}_{(2)}=0, \tilde{n}_{(3)}=-1$ and $x=y=z$, there is a $u(1)$ symmetry left which involves both dual colour and dual hypercharge, but this symmetry is not the dual to electromagnetism and has in fact no particular physical significance. Rather, we want here dual colour to be completely broken, for which case there is a wide choice. 
For lack of any other guidance, we choose to work with the simplest case when all $\tilde{n}_{(a)}$ 's are the same and equal to, say, $\tilde{n}$. In particular, we focus on $\tilde{n}=-1$ giving the common dual hypercharge of all $\tilde{\phi}^{(a)}$ as $-2 \tilde{g}_{1} / 3$, for, according to the result (2.3) of our analysis in Section 2, this is the smallest dual hypercharge a monopole of $U_{2,3}$ can have which is at the same time a dual colour triplet and a dual weak isospin singlet as the Higgs fields $\tilde{\phi}^{(a)}$ are supposed to be. In that case, the mass matrix for the gauge bosons $\tilde{C}_{\mu}^{\alpha}, \alpha=3,8,0$ reads as:

$$
\left(\begin{array}{ccc}
\frac{\tilde{g}_{3}^{2}}{4}\left(x^{2}+y^{2}\right) & \frac{\tilde{g}_{3}^{2}}{4 \sqrt{3}}\left(x^{2}-y^{2}\right) & -\frac{\tilde{g}_{3} \tilde{g}_{1}}{3}\left(x^{2}-y^{2}\right) \\
\frac{\tilde{g}_{3}^{2}}{4 \sqrt{3}}\left(x^{2}-y^{2}\right) & \frac{\tilde{g}_{3}^{2}}{12}\left(x^{2}+y^{2}+4 z^{2}\right) & -\frac{\tilde{g}_{3} \tilde{g}_{1}}{3 \sqrt{3}}\left(x^{2}+y^{2}-2 z^{2}\right) \\
-\frac{\tilde{g}_{3} \tilde{g}_{1}}{3}\left(x^{2}-y^{2}\right) & -\frac{\tilde{g}_{3} \tilde{g}_{1}}{3 \sqrt{3}}\left(x^{2}+y^{2}-2 z^{2}\right) & \frac{4 \tilde{g}_{1}^{2}}{9}\left(x^{2}+y^{2}+z^{2}\right)
\end{array}\right) .
$$

This mass matrix is a little messy to diagonalize and it is not particularly illuminating algebraically. We shall thus do so only when dealing later with numerical results. Here, we need only note that since the $\widetilde{s u}(3) \times \tilde{u}(1)$ symmetry is here completely broken, all the associated 9 gauge bosons will acquire nonzero masses.

We have argued already in the Introduction that these dual colour and dual hypercharge gauge bosons can exist as freely propagating particles. Their masses are unknown so long as the vacuum expectation values $x, y, z$ of the Higgs fields $\tilde{\phi}^{(a)}$ remain undetermined parameters. Presumably, however, the masses will be high, at least in the $\mathrm{TeV}$ range, for otherwise the bosons would have already been found. Apart from possibly being observed directly as particles in future, they can also be exchanged between dual colour and dual hyper-charges, if such exist - and we shall be considering this possibility in the next section - giving rise to interactions between them. The fact, however, that these gauge bosons are supposed to represent just the same degrees of freedom as the colour gluons $C_{\mu}$ and the hypercharge potential $B_{\mu}$ makes the physical effects of their exchange a little hard to envisage. We suggest the following picture.

Consider first a pure dual colour charge of strength $\tilde{g}_{3}$. It can interact with a similar charge via the exchange of a dual gluon $\tilde{C}_{\mu}$. In general, we recall, the colour 0 and 8 components of $\tilde{C}_{\mu}$ will mix with one another and with 
the dual hypercharge potential $\tilde{B}_{\mu}$ and will not remain thus a physical state, but for simpler presentation, let us pretend in this dicussion that this does not happen. The interaction looks like then that it will have a short range of the order of the inverse dual gluon mass which we have already stipulated to be large. However, $\tilde{C}_{\mu}$ is supposed to represent just the same degree of freedom as the colour potential $C_{\mu}$. Indeed, through the dual transform (1.1), one has in principle an explicit procedure, though a very complicated one, for constructing $C_{\mu}$ from $\tilde{C}_{\mu}$. In particle language, this would seem to mean that a dual gluon can transform itself, or "metamorphose", into a gluon. Will this then affect our conclusion above about the range of the interaction? We think not, for the gluon, though massless, is confined and cannot propagate in free space, so that the range of the interaction will still be charaterised by the mass of the dual gluon. In particular, at energies low compared with that mass scale, the interaction will be strongly suppressed by the dual gluon propagator, in the same way that weak interactions historically were considered "weak" in spite of its sizeable coupling $g_{2}$ because of its suppression at low energy by the "large" $W$ boson mass.

What will happen, however, to the interaction between dual hypercharges? They will couple with strength $\tilde{g}_{1}$ to the dual hypercharge potential $\tilde{B}_{\mu}$ which is also massive, and so it looks as if the interaction will again be short-ranged. This, however, need not be the case for $\tilde{B}_{\mu}$, like the dual gluon above, may also metamorphose into a $B_{\mu}$, but, in contrast to the colour case, $B_{\mu}$ has, via electrowek mixing, a component in the photon which is not confined and can propagate in free space. It seems to us therefore that it is eventually the photon that will govern the range of the interaction, but that the effective coupling is reduced from the original $\tilde{g}_{1}$ to $\tilde{g}_{1} / M_{\tilde{g}_{1}}^{2}$ where $M_{\tilde{g}_{1}}$ is a measure of the $\tilde{B}_{\mu}$ mass. We have in mind a picture as that represented symbolically in Figure 1, where a wavy line represents $\tilde{B}_{\mu}$, a dotted line a photon, and a little circle some sort of "metamorphosis" vertex. In other words, we are suggesting that perhaps, even though magnetic charges may exist as dual hypercharges and as such can still interact via long-ranged Coulomb-like forces, the effective strength of their interaction is drastically reduced from that expected from the Dirac quantization value. 


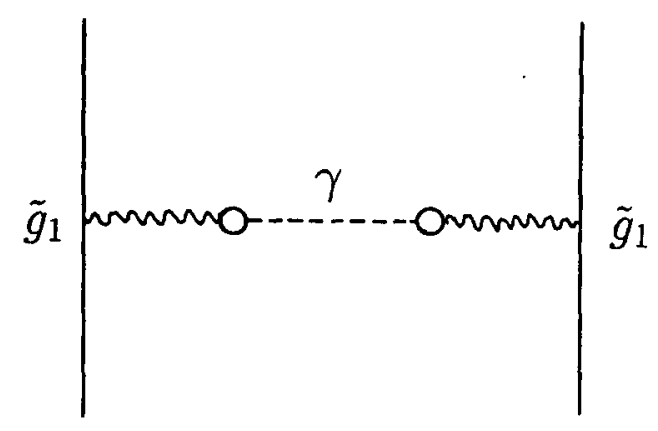

Figure 1: Interaction of dual hypercharges

Later on, we shall attempt to assign dual colour and dual hypercharges to existing particles, in which case, for the assignment to make physical sense, it will be essential to avoid having unwanted forces between the particles arising out of these dual charges. If the picture given above for the interaction of dual charges is correct, then one sees that the embarrassment can be avoided by supposing sufficiently large masses for the dual colour and dual hypercharge gauge bosons, which is possible so long as the vacuum expectation values $x, y, z$ of the Higgs fields can be freely chosen.

\section{Dual Colour as Generation Index}

The attractiveness to us of making dual colour into the generation index is twofold. On the one hand, dual colours is 3 in number, just like generations, and being there already in the gauge theory, it would be surprising, as Dirac said of monopole charges when he first discovered them, that Nature should make no use of it. Besides, if our interpretation in the previous sections were correct, dual colour would in any case manifest itself in a number of new phenomena, and if it is not as generation then it has to be otherwise accommodated. On the other hand, from the historical point of view, generation appears in the standard model just as an empirical concept introduced to fit experiment. As such it sticks out uncomfortably in a theory which is otherwise quite geometrical, and demands from us some understanding of its 
theoretical origin.

By dual symmetry, a gauge theory can be described equally in terms of either the gauge potential or its dual. In the usual description of the standard model in terms of the colour potential $C_{\mu}$, dual colour charges appear as monopoles. In Section 2, we have already analysed what colour monopoles may occur. Our first task therefore, in attempting to interpret generations as dual colour, is to assign each particle occuring in Nature a place in the table of permissible colour monopoles. In the preceding section, we have effectively done so already for the gauge bosons and the Higgs fields. We shall try now to do the same for the fermion fields.

For the moment, let us ignore weak isospin. Each fermion then, whether quark or lepton, occurs in 3 generations. If we wish to identify generation with dual colour, then it would be natural to assign the fermions to dual colour triplets, which according to Section 2, are permissible to colour monopoles. Not both the left- and right-handed fermions, however, can be assigned to dual colour triplets, for otherwise we would not be able to construct a Yukawa coupling of the fermions with the Higgs fields $\tilde{\phi}^{(a)}$ introduced above which are themselves dual colour triplets. Taking then a hint from the Salam-Weinberg theory, let us make the left-handed fermions dual colour triplets but give no dual colour charges at all to right-handed fermions, thus: $\left(\psi_{L}\right)_{a \bar{a}},\left(\psi_{R}\right)_{a}^{[b]}$ for quarks and $\left(\psi_{L}\right)_{\tilde{a}},\left(\psi_{R}\right)^{[b]}$ for leptons, where the index $a$ denotes colour, $\tilde{a}$ dual colour, both running from 1 to 3 , while the index $[b]$, though also running from 1 to 3 , is just a label for 3 types of dual colour neutral right-handed fermion fields. With this choice of dual colour for fermions, we can then write the Yukawa coupling as:

$$
\sum_{[b]} Y_{[b]} \sum_{(a)}\left(\bar{\psi}_{L}\right)^{a \bar{a}} \tilde{\phi}_{\bar{a}}^{(a)}\left(\psi_{R}\right)_{a}^{[b]}
$$

for quarks, and a similar one for leptons, where we have maintained again, as in the Higgs potential and in the kinetic energy of the Higgs fields, a symmetry between $\tilde{\phi}^{(a)}$ for different $(a)$.

The mass matrix $m$ for fermions is to be obtained by inserting for $\tilde{\phi}^{(a)}$ in 
(5.1) their vacuum expectations given in (4.4), giving:

$$
m=\left(\begin{array}{ccc}
x a & x b & x c \\
y a & y b & y c \\
z a & z b & z c
\end{array}\right)=\left(\begin{array}{l}
x \\
y \\
z
\end{array}\right)(a, b, c)
$$

where we have written $a=Y_{[1]}, b=Y_{[2]}, c=Y_{[3]}$ for short. For this we obtain, for $\rho^{2}=\left(|a|^{2}+|b|^{2}+|c|^{2}\right)$ :

$$
m m^{\dagger}=\rho^{2}\left(\begin{array}{ccc}
x^{2} & x y & x z \\
y x & y^{2} & y z \\
z x & z y & z^{2}
\end{array}\right)=\rho^{2}\left(\begin{array}{l}
x \\
y \\
z
\end{array}\right)(x, y, z)
$$

which being factorizable as shown, is a matrix of rank 1 , having thus only one nonzero eigenvalue $\rho^{2} \zeta^{2}$, with $\zeta^{2}=x^{2}+y^{2}+z^{2}$. The matrix $\mathrm{mm}^{\dagger}$ can be diagonalized by:

$$
U=\left(\begin{array}{ccc}
\alpha x & \alpha y & \alpha z \\
\beta / x & \beta \omega^{2} / y & \beta \omega / z \\
\gamma\left[\frac{y \omega^{2}}{z}-\frac{z \omega}{y}\right] & \gamma\left[\frac{z}{x}-\frac{x \omega^{2}}{z}\right] & \gamma\left[\frac{x \omega}{y}-\frac{y}{x}\right]
\end{array}\right)
$$

with:

$$
\begin{aligned}
\alpha^{-2} & =x^{2}+y^{2}+z^{2} \\
\beta^{-2} & =\frac{1}{x^{2}}+\frac{1}{y^{2}}+\frac{1}{z^{2}} \\
\gamma^{-2} & =\frac{1}{x^{2} y^{2} z^{2}}\left(x^{2} y^{4}+y^{2} z^{4}+z^{2} x^{4} x^{4} y^{2}+y^{4} z^{2}+z^{4} x^{2}+3 x^{2} y^{2} z^{2}\right) \\
& =\alpha^{2} \beta^{-2}
\end{aligned}
$$

and $\omega=\exp (2 \pi i / 3)$ a cube root of unity. Thus:

$$
U m m^{\dagger} U^{\dagger}=\operatorname{diag}\left(\rho^{2} \zeta^{2}, 0,0\right) .
$$

We conclude therefore that all the mass in this mass matrix is soaked up by one single massive state, leaving the other 2 massless. Furthermore, since the diagonalizing matrix $U$ in (5.6) depends only on the vacuum expectation values $x, y, z$ of the Higgs fields $\tilde{\phi}^{(a)}$ which are themselves independent of 
which fermions they are coupled to, it follows that $U$ must be the same for $u$-type and $d$-type quarks, giving thus the identity matrix as the CabbiboKobayashi-Moskawa (CKM) matrix. Now, although such a mass matrix is highly degenerate, it is not at all bad as a first approximation to the physical situation, given that for both $u$-type and $d$-type quarks and also for leptons, the empirical masses for the 2 lower generations are in every case no more than 6 percent of the highest generation mass, while the empirical CKM matrix has its diagonal elements all differing from unity by at most 3 percent and its largest off-diagonal element of order only 20 percent. [23] Indeed, these significant empirical facts are a bit of a mystery in conventional formulations of the standard model, having there no obvious explanation, and we regard it as an attractive feature of our scheme that it should lead immediately to such a sensible zeroth order approximation. In the next section, we shall consider the means whereby the above degeneracy at zeroth order may be lifted perturbatively to give nonvanishing values for the lower generation masses and for the off-diagonal CKM matrix elements. Here we only note that the masses of the highest generation, namely $t, b$ and $\tau$, can of course be fitted to the experimental values by adjusting the Higgs fields vacuum expectation values $x, y, z$ and the Yukawa couplings $a, b, c$.

Obviously, the great danger in interpreting generations as a broken gauge symmetry is that gauge symmetries imply gauge interactions, and none has been observed between generations besides the usual colour and electroweak (and of course gravitational) forces. This is particularly worrying with dual colour, for the gauge interactions here are in principle strong. Thus, for example, the neutrinos, which carry a generation index, and hence in the present scheme also dual colour, can in principle interact strongly with one another, which would be far from the truth as we now know it. However, as already pointed out in the last section, dual colour is broken, with all gauge bosons acquiring masses. The effect of their exchange is therefore suppressed by their propagators at energies low compared with their masses. Thus, by choosing the gauge boson masses sufficiently high, one can in principle always reduce the gauge interaction due to dual colour sufficiently to keep within experimental bounds. For example, a crude estimate shows that 
by choosing dual gluon masses greater than $1 \mathrm{TeV}$, we can make the dual colour interactions between neutrinos in the present scheme weaker than the standard weak interactions between them. Now, from (4.5) and (4.6), one sees that one can make dual gluon masses as large as one likes so long as the vacuum expectation values $x, y, z$ of the Higgs fields $\tilde{\phi}^{(a)}$ remain unconstrained. At the same time, one sees from (5.6) that one can still keep the quark masses at around the experimental scale by adjusting appropriately the Yukawa couplings $a, b, c$. This is the tactic we shall advocate, which is at least possible when we are treating the breaking of dual colour in isolation from the breaking of weak isospin as we have been doing so far in this section.

However, in combining the treatment of symmetry breaking for both dual colour and weak isospin, we meet with a problem. The left-handed fermion is not only a triplet of dual colour but also a doublet of weak isospin, thus: $\left(\psi_{L}\right)_{r}^{a \tilde{a}}$ for quarks and $\left(\psi_{L}\right)_{r}^{\tilde{a}}$ for leptons while the right-handed fermion is a singlet in both. Thus, given that our Higgs fields $\tilde{\phi}^{(a)}$ and $\phi^{(r)}$ carry each only dual colour or weak isospin, we would need both to build an invariant coupling with the fermion fields, e.g. for quarks:

$$
\sum_{[b]} Y_{[b]} \sum_{(a)}\left(\bar{\psi}_{L}\right)_{r}^{a \tilde{a}} \tilde{\phi}_{\bar{a}}^{(a)} \phi^{(1) r}\left(\psi_{R}\right)_{a}^{[b][1]}+\sum_{[b]} Y_{[b]}^{\prime} \sum_{(a)}\left(\bar{\psi}_{L}\right)_{r}^{a \tilde{\tilde{\phi}}} \tilde{\phi}_{\bar{a}}^{(a)} \phi^{(2) r}\left(\psi_{R}\right)_{a}^{[b][2]}
$$

where the indices [1] and [2] denote the 2 types of right-handed isosinglets with hypercharge respectively $2 / 3$ and $-1 / 3$. This is not properly a Yukawa coupling and looks like being nonrenormalizable.

If we expand the Higgs fields $\tilde{\phi}$ and $\phi$ about their vacuum values, we would obtain the mass matrices of the $u$-and $d$-type quarks respectively as:

$$
\begin{gathered}
m_{u}=v\left(\begin{array}{l}
x \\
y \\
z
\end{array}\right)(a, b, c), \\
m_{d}=w\left(\begin{array}{l}
x \\
y \\
z
\end{array}\right)\left(a^{\prime}, b^{\prime}, c^{\prime}\right),
\end{gathered}
$$

and Yukawa-type coupling to the oscillations $\tilde{\phi}^{\prime}$ and $\phi^{\prime}$ of the Higgs fields 
about their respective vacuum expectations, thus:

$$
\sum_{[b]} Y_{[b]} \sum_{(a)}\left(\bar{\psi}_{L}\right)_{r}^{a \tilde{a}}\left(\tilde{\phi}^{\prime}\right)_{\tilde{a}}^{(a)} v\left(\psi_{R}\right)_{a}^{[b][1]}+\left(\bar{\psi}_{L}\right)_{r}^{a \tilde{a}} v^{-1} m_{\tilde{a}[b]}\left(\phi^{\prime}\right)^{(1) r}\left(\psi_{R}\right)_{a}^{[b][1]}
$$

for the $u$-type quarks, and a similar expression for the $d$-type quarks. The mass matrices (5.8) and (5.9) are of the form we wanted in (5.2) apart from a different normalization convention. The Yukawa couplings to the fields $\tilde{\phi}^{\prime}$ and $\phi^{\prime}$ are also as expected, with the second term being the familiar coupling to the Higgs field of standard electroweak theory, and the first term being a coupling for the dual colour Higgs that we can accept. There will be, however, a further term in the expansion in which both $\tilde{\phi}^{\prime}$ and $\phi^{\prime}$ occur which, though arguably small for oscillations small compared with their vacuum expectation values, can nevertheless make the theory nonrenormalizable.

We have considered 2 ways of addressing this problem. The first is to combine the 2 sets of Higgs fields $\tilde{\phi}_{\bar{a}}^{(a)}$ and $\phi_{r}^{(r)}$ into a single set, say, $\Phi_{\bar{a} r}^{(a)(r)}$, carrying both dual colour and weak isospin. In that case, we can write down a genuine Yukawa coupling for our fermions as follows:

$$
\sum_{(a)[b](r)[s]} Y_{[b][s]}\left(\bar{\psi}_{L}\right)^{a \bar{a} r} \Phi_{\bar{a} r}^{(a)(r)}\left(\psi_{R}\right)_{a}^{[b][s]}
$$

The disadvantage, however, is that the breaking of dual colour and weak isospin will then be governed by the same vauum expectation values of these Higgs fields, and hence would occur at comparable energy scales. It would thus remove the freedom of pushing the dual colour gauge bosons to high masses so as to suppress unwanted interactions between generations as we had advocated above. If one takes this route, therefore, one will have to find some other cleverer way for suppressing the unwanted interactions to within experimental bounds, which though perhaps possible seems to us somewhat contrived and difficult to achieve.

The alternative that we prefer which leaves free the symmetry breaking scale for dual colour compared with that for weak isospin is to accept (5.7) but to regard the present scheme as just a low energy effective theory and some of the fields we have so far listed as composites of some yet undiscovered more fundamental fields. Within the present dual framework, there is good reason 
to suspect that that may indeed be the case. Dual symmetry implies that the electroweak $s u(2)$ symmetry should have a dual, i.e. an $\widetilde{s u}(2)$ symmetry. At the fundamental level, therefore, one expects that Higgs fields (frames) and fermion fields should carry also $\widetilde{s u}(2)$ indices. Up to now, however, we have considerd only $\widetilde{s u}(2)$ singlets which are all that is required so far to accommodate the known particle spectrum. The rationale for that, we suggest, is that the electroweak $s u(2)$ symmetry being broken and Higgsed, 't Hooft's argument[10] would imply that its dual $\widetilde{s u}(2)$ should be unbroken and confined. In that case, only $\widetilde{s u}(2)$ singlets can exist in the free state, which are all that one has seen at present, and unless one can perform deep inelastic experiment at high enough energy, one would not be able to see their $\widetilde{s u}(2)$ internal structure. One can even argue that, the $\widetilde{s u}(2)$ coupling $\tilde{g}_{2}$, as estimated from the experimental value of $\alpha_{2}=g_{2}^{2} / 4 \pi \sim 0.033$ and the Dirac quantization condition (2.11), being more than 10 times larger than the $s u(3)$ colour coupling $g_{3}$, the confinement by dual weak isospin would be much deeper than by colour and would require much higher energy to detect. Now, if some of the "fundamental" particles we know are in fact composites, it would not be surprising if some of their couplings, in particular the "Yukawa" coupling (5.7), appear nonrenormalizable. It is not easy, of course, to guess the fundamental fields and couplings at the deeper level, but is is not hard to find examples which can give rise to the effective coupling (5.7) we want. The construction in Figure 2 is a possibility, in which each line is labelled by the indices it carries, $a$ being colour, $\tilde{a}$ dual colour, $r$ weak isospin, $\tilde{r}$ dual weak isospin, and the last number dual weak hypercharge. Each line in Figure 2 is an admissible combination of dual charges as listed in (2.3) as it ought to be. The first (from left) and last fermion lines are the $\psi_{L}$ and $\psi_{R}$ above, the first Higgs line is $\phi^{r}$, while the second and third Higgs lines are supposed to be confined together by their dual weak isospin as indicated by $\tilde{r}$ to form the other Higgs fields $\tilde{\phi}^{\tilde{a}}$ as composites. If the remaining fermion lines are assumed to be heavy, we would obtain (5.7) as an effective coupling.

For the present, we leave the choice of the 2 alternatives open as it will make no difference yet to our phenomenology, although in the considerations which follow, our thinking may have been biassed towards the second choice. 


\begin{tabular}{|c|c|c|c|}
\hline$a, \tilde{a}, r, 2 / 3$ & $a, \tilde{a}, 2 / 3$ & $a, \tilde{a}, \tilde{r},-1 / 3$ & $a, 0$ \\
\hline \multirow[t]{2}{*}{$L$} & $R$ & $L$ & $R$ \\
\hline & $r, 0$ & $\tilde{r},-1$ & $\tilde{a}, \tilde{r}, 1 / 3$ \\
\hline
\end{tabular}

Figure 2: Example of an effective coupling

\section{CKM Matrix and Masses for Lower Gen- erations}

Although zero masses for lower generations and the identity matrix as the CKM matrix are reasonable as zeroth order approximations, one would need of course to envisage some mechanism whereby this degenerate scenario can be lifted so as to give eventually more realistic values for these parameters. Within the framework of the standard model, loop corrections are an obvious possibility. However, the fermion mass matrix here being at zeroth order factorizable as in (5.2), loop corrections are quite restricted in property and it is not obvious at first sight that they are capable of performing that function. What we wish to show now is that they can indeed do so, at least in principle, although whether they will actually give the correct answers to fit with experiment can only be decided by detailed calculations.

Some one-loop corrections to the fermion mass matrix are depicted in Figure 3, where a full line represents fermions, a wavy line gauge bosons, and a dotted line Higgses. However, even before performing any calculation, one can see that these corrections will not alter the factorized form of the 


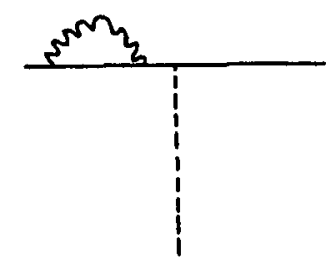

(a)

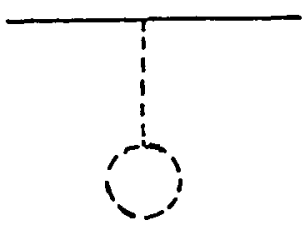

(c)

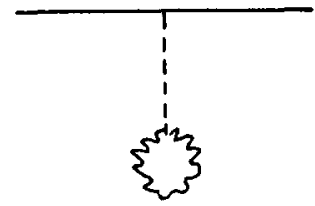

(d)

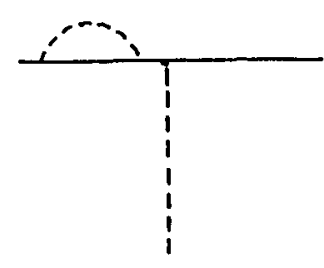

(b)

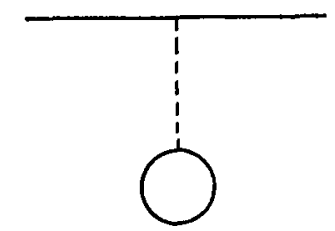

(e)

Figure 3: One loop corrections to the fermion mass matrix

mass matrix. Diagrams (a) and (b) will only premultiply the factorized zeroth order mass matrix by another matrix so that the result has to remain in the factorized form. On the other hand, diagrams (c), (d), and (e) are linear combinations of matrices all of the factorized form (5.2) with the same parameters $a, b$ and $c$ so that the result is again factorized.

A "vertex renormalization" diagram of the type shown in Figure 4(a) can in principle break factorizability but in the present framework, such diagrams do not exist. Since only the left-handed fermions here carry nonabelian charges ( 3 for dual colour and $\mathbf{2}$ for weak isospin) with the righthanded fermions neutral under both these symmetries, the corresponding gauge bosons (namely the dual gluons $\tilde{C}_{\mu}^{\bar{a}}$, and the weak bosons $W_{\mu}^{r}$ ) couple only to $\psi_{L}$, not to $\psi_{R} . U(1)$ and $\tilde{U}(1)$ gauge bosons can couple to both $\psi_{L}$ and $\psi_{R}$, depending on their $U(1)$ and $\tilde{U}(1)$ charges, but these however do not rotate the generation (i.e. dual colour) indices, leaving thus the factorized form of the mass matrix still intact. On the other hand, although the diagram Figure 4(b) with a Higgs loop does exist since the Higgs couples $\psi_{L}$ to $\psi_{R}$ as shown, the diagram has a factorized Yukawa coupling matrix on the extreme left and right, and must therefore remain in the factorized form. 


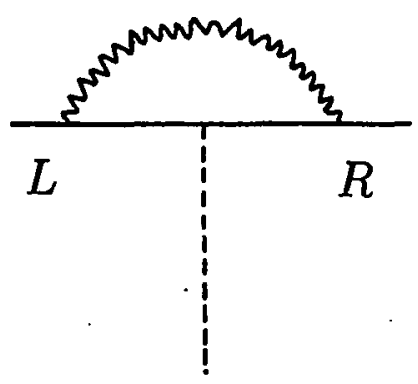

(a)

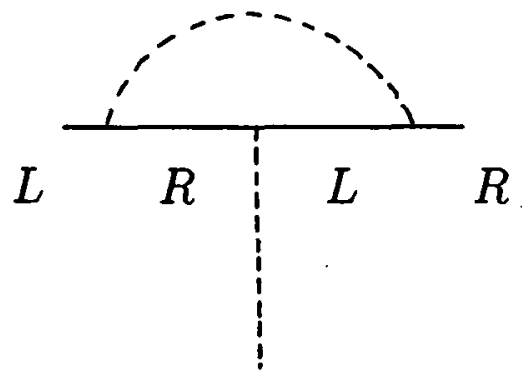

(b)

Figure 4: Vertex renormalizations to the fermion mass matrix

The above analyis can be extended to diagrams with higher loops. For basically the same reasons as those given above for one-loop diagrams, it can be seen that even higher loop diagrams will find it hard to break the factorizability of the mass matrix, and indeed we have not found a single one capable of doing so. We are thus forced to accept that, barring nonperturbative effects, the factorized form of the mass matrix will remain intact to all orders.

The fact that the mass matrix should remain factorized, however, does not necessarily mean that loop corrections can never lift the degeneracy at the zeroth order. Take, for example, the dual gluon loop diagram of Figure $3(a)$. Although it cannot break the factorizability of $m$, it will in general rotate its left-hand factor, thus:

$$
m_{0}=\left(\begin{array}{c}
x \\
y \\
z
\end{array}\right)(a, b, c) \longrightarrow m_{1}=\left(\begin{array}{c}
x_{1} \\
y_{1} \\
z_{1}
\end{array}\right)(a, b, c) .
$$

The amount of this rotation will depend on the parameters in the original zeroth order mass matrix. In particular, these parameters being different for the $u$-type and $d$-type quarks, for example, the resultant left-hand factor $\left(x_{1}, y_{1}, z_{1}\right)$ after the one-loop correction will be different for $u$ and $d$. It follows then that the matrices $U$ and $U^{\prime}$ diagonalizing $m_{1} m_{1}^{\dagger}$ respectively for 
$u$ and $d$ as given in (5.4) will also be different, giving thus a nontrivial (i.e. non-identity) CKM matrix $V=U U^{\prime-1}$. We notice, however, that this will happen only when the vacuum expectation values $x, y, z$ of the Higgs fields are different.

As the mass matrix remains factorizable after loop corrections, it is still of rank 1 and have thus still only 1 non-zero eigenvalue. It might then appear that the 2 lower generations will still have vanishing mass. However, this need not be the case, for loop corrections, apart from rotating the mass matrix as in (6.1), also make it run by virtue of the renormalizing group equation, and when the mass matrix changes its value depending on the energy scale at which it is measured, it is not immediately clear how the actual masses of particles ought to be defined. When considering only one particle, the conventional wisdom is that the running squared mass $m^{2}\left(Q^{2}\right)$ has to be evaluated at a value of $Q^{2}$ equal to its own value at that $Q^{2}$, which is then designated as the mass of the particle. When we are dealing with a mass matrix of the factorized form (5.2), however, or indeed with any matrix of rank 1 , it is not so obvious what the proper procedure to define particle masses ought to be. We suggest the following.

Given that loop corrections are not supposed to break the factorized form of the fermion mass matrix (5.2), it will remain of rank 1 at all energy scales so that the eigenstate with the highest eigenvalue can always be defined without any difficulty. The other 2 states with zero eigenvalues, however, are indistinguishable. Imagine then that the mass matrix is run via the renormalization group equation from a high energy scale down. At every scale, we can diagonalize the matrix and identify the eigenstate with the nonzero eigenvalue. Let us then run the scale down until this eigenvalue takes on the same value as the scale at which it is evaluated. Recalling the conventional wisdom cited above for defining the mass in the case of a single particle, we can then legitimately define this value as the mass of the highest generation fermion. At this energy, of course, as indeed at any energy, since the mass matrix remains of rank 1 , the other 2 eigenvalues are zero, but they should not be interpreted as the masses of the 2 lower generations, for they are evaluated at the wrong scale. To find the actual masses, the mass matrix 
should be run further down in scale and evaluated at the masses of the lower generations, whatever these may be. We have now to specify exactly how this ought to be done.

The identification of the highest generation state at its mass scale also specifies a 2-dimensional subspace of states orthorgonal to the highest generation, namely the eigen-subspace with zero eigenvalues in this case. It is clear that the state vectors of the 2 lower generations, being independent physical entities to the first, should lie in this subspace. Let us now run the mass matrix down to a lower scale. We have seen already that loop corrections can rotate the left factor of the mass matrix, so that, in general, the $\mathrm{mm}^{\dagger}$ matrix that we have diagonalized at the highest generation mass will no longer remain diagonal at the lower energy. We can of course rediagonalize the matrix at the lower energy obtaining again 1 nonzero and 2 zero eigenvalues, but the diagonalizing matrix at the lower energy will not be the same as that at the mass of the highest generation we have obtained before. In other words, the 2-dimensional subspace we have identified before at the highest generation mass scale as containing the states of the 2 lower generations will no longer lie within the eigen-subspace of eigenvalue 0 at the lower energy. To be specific, suppose we call the eigenvector for the highest generation $\mathbf{v}_{1}$ and define 2 other mutually orthogonal (normalized) vectors $\mathbf{v}_{2}$ and $\mathbf{v}_{\mathbf{3}}$ orthogonal also to $v_{1}$, all at the mass scale of the highest generation, then the mass submatrix:

$$
<\mathbf{v}_{i}|m| \mathbf{v}_{j}>; i, j=2,3,
$$

will in general be nonzero at the lower energy scale to which it is run. But this, according to the preceding arguments, has to be interpreted as the mass submatrix for the 2 lower generations.

The $2 \times 2$ matrix (6.2), being a nonzero sub-matrix of the rank 1 matrix $m$, is of course still rank 1 , so that it can also be diagonalized at every energy giving 1 nonzero eigenvalue and the other zero. We can then repeat the above procedure and run the mass matrix on down via the renormalization group equation until the nonzero eigenvalue of (6.2) equals the scale at which it is evaluated. This value, in conformity with what has gone before, we should define as the mass of the second highest generation and is, of course, nonzero. 
The diagonalization of the matrix (6.2) at the second generation mass identifies in turn the eigenvector with the nonzero eigenvalue as the state vector of the second generation. Let us call this vector $\mathbf{v}_{2}^{\prime}$, which is by definition orthogonal to $v_{1}$, the state vector for the highest generation, and in general different from $\mathbf{v}_{\mathbf{2}}$. Further, we can define the remaining eigenvector with zero eigenvalue $\mathbf{v}_{3}^{\prime}$ as the state vector of the lowest generation, and it is by construction orthogonal to both $\mathbf{v}_{\mathbf{1}}$ and $\mathbf{v}_{2}^{\prime}$ as it should be. At the mass scale of the second generation, of course, the quantity $(1 \times 1$ submatrix $)$ :

$$
<\mathbf{v}_{3}^{\prime}|m| \mathbf{v}_{3}^{\prime}>
$$

vanishes, but as before, this should not be interpreted as the mass of the lowest generation fermion since it is evaluated at the wrong scale. We have again to run it down further via the renormalization group equation for $m$ until the value of (6.3) equals the scale at which it is evaluated. At that scale, $\mathbf{v}_{3}^{\prime}$ will not in general lie within the eigen-subspace of $m$ with zero eigenvalue, so that (6.3) can be nonvanishing, or that the lowest generation fermion also will have nonzero mass. Since at each stage, the leading remaining generation soaks up all the mass in the matrix, leaving the next generation to acquire only whatever mass it can by running, the mass will go down by a large factor from each generation to the next, qualitatively the same as what experiment is telling us.

One sees therefore that although the mass matrix remains factorizable and of rank 1 after loop corrections, the effects of the corrections will nevertheless be sufficient to give nonzero masses to the lower generation fermions and to make the CKM matrix deviate from the identity. However, whether these effects can be made to give numbers close to the experimental values by adjusting the free parameters still remaining in the scheme is a question that can only be answered by a detailed calculation, which we have begun but are far from being in a position yet to report on. We can at present only give the following two trial calculations as illustrations for the sort of effects we shall get.

As illustration for loop corrections to the identity CKM matrix, let us consider for dual colour the 1-loop diagrams listed in Figure 3 which have 
already been evaluated by Weinberg [24] in a general Yang-Mills-Higgs framework. He gave the answer as a sum of 5 terms, of which the last 2 due to Higgs loops and tadpoles, called $\Sigma_{e f f}^{\phi 1}$ and $\Sigma_{e f f}^{T 1}$ by him, depend on the Higgs boson mass matrix of which we have yet insufficient knowledge. The other 3 terms, depending on the Higgs fields' vacuum expectation values but not on their masses, we can in principle evaluate modulo some unknown parameters and ambiguities that we shall make clear. Furthermore, the term called $\Sigma_{e f f}^{A T}$ by Weinberg rotates the fermion mass matrix $m$ the same way for $u$-type and $d$-type quarks, whereas in order to contribute to the CKM matrix, a loop correction has to rotate $m$ differently for $u$ and $d$. There remain then only 2 terms which affect the CKM matrix directly for us to consider, namely:

$$
\begin{aligned}
\Sigma_{e f f}^{A 1}= & \frac{1}{16 \pi^{2}} \sum_{N} \int_{0}^{1} d x\left[-2 m_{W} \bar{t}_{N}(1-x)+4 \gamma_{4} \bar{t}_{N} \gamma_{4} m_{W}\right] \ln \left(\mu_{N}^{2}+\frac{m_{W}^{2} x^{2}}{1-x}\right)_{(6.4)}, \\
\Sigma_{e f f}^{A \phi}= & \frac{1}{16 \pi^{2}} \sum_{N} \frac{1}{\mu_{N}^{2}} \int_{0}^{1} d x\left\{(1-x) m_{W}\left[\gamma_{4} m_{W}, \bar{t}_{N}\right] \gamma_{4}+\gamma_{4}\left[\gamma_{4} m_{W}, \bar{t}_{N}\right] m_{W}\right\} \\
& \left\{\ln \left(\frac{m_{W}^{2} x^{2}}{1-x}\right)-\ln \left(\mu_{N}^{2}+\frac{m_{W}^{2} x^{2}}{1-x}\right)\right\} \gamma_{4}\left[\gamma_{4} m_{W}, \bar{t}_{N}\right],
\end{aligned}
$$

where $\mu_{N}$ are the masses of the dual colour and dual hypercharge gauge bosons, namely those listed in (4.5) together with the eigenvalues of the mass matrix in (4.6). The fermion mass matrix used here is:

$$
m_{W}=\frac{\rho}{\zeta}\left(\begin{array}{l}
x \\
y \\
z
\end{array}\right)(x, y, z)
$$

which is, crudely speaking, the square root of the matrix $m m^{\dagger}$ in (5.3). The couplings $\bar{t}_{N}$ are defined as:

$$
\begin{aligned}
& \bar{t}_{N}=-\frac{\tilde{g}_{3}}{2} \lambda_{N} \frac{1}{2}\left(1-\gamma_{5}\right), \quad N=1,2,4,5,6,7 \\
& \bar{t}_{N}=\left\{-\frac{\tilde{g}_{3}}{2} \lambda_{3} C_{3 N}-\frac{\tilde{g}_{3}}{2} \lambda_{8} C_{8 N}+\frac{2}{3} \tilde{g}_{1} C_{0 N}\right\} \frac{1}{2}\left(1-\gamma_{5}\right), N=3,8,0,(6.7)
\end{aligned}
$$


with $C$ being the matrix which diagonalizes (4.6) and $\lambda_{N}, N=1, \ldots, 8$ the Gell-Mann matrices.

Apart from the coupling constants $\tilde{g}_{3}$ and $\tilde{g}_{1}$ which can be determined from the experimental values of their duals $g_{3}=\sqrt{4 \pi \alpha_{3}}$ and $g_{1}=\sqrt{4 \pi \alpha_{1}}$ via the Dirac quantization conditions (2.5) and (2.11), the expressions in (6.4) and (6.5) depend on the vacuum expectation values of the Higgs fields $x, y, z$ and on the Yukawa couplings $a, b, c$ through $\rho$, as defined in section 5. The parameters $x, y, z$ are unknown, but once these are given then $\rho$ can in principle be determined by normalizing $\rho \zeta$, the nonzero eigenvalue of $m$, on the experimental mass of the highest generation fermion, namely $m_{\text {top }}$ and $m_{\text {bottom }}$ for respectively the $u$-type and $d$-type quarks. In practice, however, there is here an ambiguity in normalizing $\rho$ for the following reason. There are terms in $\Sigma_{\text {eff }}^{(A 1)}$ as well as in the other Weinberg terms that we have dropped which are scale dependent, and though either not rotating $m_{W}$ at all, or else rotating $m_{W}$ the same way for $u$ - and $d$-type quarks, and so not affecting the CKM matrix directly, nevertheless changes the normalization of $m_{W}$. This is presumably related ultimately to the running of these quantities with changing scales which we have not yet sorted out fully. As a result, we have to treat $\rho$ also as a parameter for the moment, and cannot fix the actual size of off-diagonal CKM matrix elements. Further, not having sorted out the running effects, we also cannot, using the method outlined earlier in this section, identify the quarks of the 2 lower generations. Hence, we cannot at present specify $V_{u s}$ and $V_{c d}$, or distinguish $V_{u b}$ from $V_{c b}$ and $V_{t d}$ from $V_{t s}$. The significance of this present exercise is thus strictly limited.

Putting in arbitrarily the parameters $x=1, y=2 / 3, z=1 / 3$, we obtained from (6.4) and (6.5) the following matrix for the absolute values of CKM matrix elements, where $\rho$ has been adjusted to give off-diagonal elements roughly of the order of a percent.

$$
\left(\begin{array}{lll}
.9998 & .0173 & .0130 \\
.0166 & .9998 & .0124 \\
.0130 & .0123 & .9998
\end{array}\right)
$$

Given the limitations stated in the preceding paragraph, the only conclusions we can draw at present are that this 'mock' CKM matrix (i) does get rotated 
from the identity by loop corrections, (ii) remains roughly though not exactly symmetric, and (iii) is in general complex, all of which are properties apparently exhibited by the actual CKM matrix obtained from experiment. [23] This is not much, but still enough perhaps as encouragement for further exploration.

As illustration for generating masses for lower generation fermions, consider the renormalization group equations usually given for the standard model [25]:

$$
\begin{aligned}
16 \pi^{2} \frac{d U}{d t} & =\frac{3}{2}\left(U U^{\dagger}-D D^{\dagger}\right) U+\left(\Sigma_{u}-A_{u}\right) U, \\
16 \pi^{2} \frac{d D}{d t} & =\frac{3}{2}\left(D D^{\dagger}-U U^{\dagger}\right) D+\left(\Sigma_{d}-A_{d}\right) D,
\end{aligned}
$$

where $U$ and $D$ are respectively the Yukawa coupling matrices to the electroweak Higgs field for respectively the right-handed $u$ - and $d$-type quarks ${ }^{5}$, and $\Sigma_{u, d}$ and $A_{u, d}$ are the Higgs self-energy and gauge boson loop contributions whose explicit forms need not here bother us.

The matrices $U$ and $D$ can of course be diagonalized at any scale, but do not remain diagonal in general on running, and what interest us for the problem at hand are just those terms which contribute towards the dediagonalization of $U$ and $D$, namely the $D D^{\dagger}$ term in (6.9) and the $U U^{\dagger}$ term in (6.10). In the basis where $U$ is diagonal, $D$ is not diagonal, and vice versa, by virtue of a nontrivial CKM matrix $V$, so that for the de-diagonalizing effects alone which interest us, we may write the renormalization group equations (6.9) and (6.10) as:

$$
\begin{aligned}
16 \pi^{2} \frac{d U}{d t} & =-\frac{3}{2} D D^{\dagger} U, \\
16 \pi^{2} \frac{d D}{d t} & =-\frac{3}{2} U U^{\dagger} D .
\end{aligned}
$$

Now in the philosophy of the present scheme, the main effect for dediagonalizing $U$ and $D$ is supposed to come from diagrams with dual colour gauge and Higgs boson loops, as already discussed above. These dual colour

\footnotetext{
${ }^{5}$ The symbol $U$ adopted here following the usual convention should not, of course, be confused with the diagonalizing matrix in (5.4).
} 
loop effects, however, have not been included in the equations (6.9) and (6.10), which indeed we do not even yet know how to calculate. However, since it was these omitted effects which are supposed to give rise to the nontrivial CKM matrix in the first place, the de-diagonalizing effects from the mixing due to the CKM matrix itself which are included in (6.11) and (6.12) would have to be regarded in this philosophy as only secondary effects induced by the primary dual colour loop contributions. Nevertheless, we think it worthwhile to study (6.11) and (6.12) as illustrations for the effects on the lower generation fermion masses that one can expect.

As we shall be interested in running the equations only over small ranges of the order of the mass differences between generations, we may take the linearized equations and consider the CKM matrix itself as constant over these ranges. Starting then with a diagonalized mass matrix at the mass scale of the highest generation, in our case $\operatorname{diag}\left(m_{t o p}, 0,0\right)$ for the $u$-type and $\operatorname{diag}\left(m_{\text {bottom }}, 0,0\right)$ for the $d$-type quarks, and running it down to lower energies, we obtain:

$$
\begin{aligned}
U_{t} & =V \operatorname{diag}\left(\exp \left[-\frac{3\left(m_{b} / w\right)^{2} t}{32 \pi^{2}}\right], 0,0\right) V^{-1} \operatorname{diag}\left(m_{t}, 0,0\right), \\
D_{t} & =V^{-1} \operatorname{diag}\left(\exp \left[-\frac{3\left(m_{t} / v\right)^{2} t}{32 \pi^{2}}\right], 0,0\right) V \operatorname{diag}\left(m_{b}, 0,0\right),
\end{aligned}
$$

where, $V$ being non-diagonal, one sees that the mass matrices, though diagonalized at the highest generation mass, will become non-diagonal when run to the lower energy, as expected.

Now in the philosophy of the present scheme, the difference in the top and bottom mass comes mainly from the the difference between $v$ and $w$, i.e. the vacuum expectation values of resepectively the Higgs fields $\phi^{(1)}$ and $\phi^{(2)}$, so that the Yukawa couplings $m_{t} / v$ and $m_{b} / w$ are comparable in magnitude. In that case, we can put:

$$
m_{t} / v \sim m_{b} / w \sim(180 \mathrm{GeV} / 246 \mathrm{GeV}) .
$$

Inserting this value in (6.13) and (6.14) above, together with the experimentally measured values of the CKM matrix elements, one obtains that 
on running from the highest generation to the next, say e.g. from the top (bottom) to the charm (strange) quark mass, the equations would generate off-diagonal elements in $U$ or $D$ of the order of $10^{-3}$ times the highest generation mass. This is not enough to explain the actual mass values of the second generation which is of the order of a few percent of the highest generation. However, one recalls that the effect represented by (6.13) and (6.14) are supposed to be only secondary effects obtained from the primary dual colour effects that we have not yet learned to calculate. If we argue naively that the factor of suppression in mass from one generation to the next due to the primary effect should be of the order of the square root of that due to the secondary effect, then the answer we obtained is about right. The above argument, for whatever it is worth, can be repeated for the suppression from the second to the lowest generation and the answer is still comparable with what is seen in experiment.

\section{Concluding Remarks}

The feature we find most attractive in the present scheme is the possibility to assign both to the Higgs fields and to the fermion generations each a natural place. This is a consequence of the recently discovered nonabelian dual symmetry[17] to the extent that the necessary niches exist because of it in the form of the transformation matrix $\omega$ and of the concept of a local dual colour symmetry. But the actual assignment of these niches to Higgs fields and to fermion generations involves of course some, perhaps somewhat daring but to us quite reasonable, assumptions and the merit or otherwise of these must rest in the end on the compatibility of their predictions with experiment.

As far as present investigations go, the scheme has scored a number of positive points, among which we count the prediction of exactly 3 generations, the mass hierarchy between them, the near identity CKM matrix, and the possibility of evaluating lower generation masses and off-diagonal CKM matrix elements perturbatively. The first three points are all significant and noted empirical facts which lack explanation in the usual formulation of the 
standard model, but seem to have found each a raison d'etre in the present scheme.

On the other hand, there are also consequences which can give rise to potential disagreement with experiment, among which the most worrying is the prediction of new interactions due to dual gluon exchange. We argued above that these are suppressed by the dual gluon propagator, and so long as these are large enough, we may not notice the interactions due to their exchange at present experimental energy. This suppression, however, has its limit, on 2 counts. First, the loop corrections, which we claimed in the preceding section may lead to nonzero off-diagonal CKM matrix elements and lower generation masses, also depend on the masses of dual gluon, and if one makes these latter masses too large, then the loop correction may be too small to explain the experimental effects. One shall then have to devise other means for lifting the zeroth order degeneracy. Secondly, even if one can make the masses of dual gluons very large, there will eventually come a point at which the propagator suppression will no longer work, and the interaction from dual gluon exchange, say e.g. between neutrinos which carry dual colour, will become very strong. Will this not violate some astronomical or cosmological bounds? We do not know. By the same token, the scheme may conceivably be in conflict with some currently held theoretical ideas on asymptotic behaviour. At first sight, it may appear that the dual colour coupling $\tilde{g}_{3}$, being inversely proportional to the usual colour coupling $g_{3}$, will grow with energy and so spoil completely such cherished concepts as asymptotic freedom. We are, however, not sure that this will be so. As already stated repeatedly above, the dual gluon $\tilde{C}_{\mu}$ does not represent a different degree of freedom to the gluon $C_{\mu}$, but should rather be regarded as a composite (a hadron!) formed from the usual colour gluons and the coloured Higgs fields. If so, their exchange should be compared not with elementary exchanges but with say pion-exchange between hadrons which do not spoil asymptotic freedom. Nevertheless, at finite energies, dual gluon exchanges will affect the running of various quantities and hence may lead to potential discrepancy with experiment.

Let us assume optimistically that the present scheme will survive these 
possible pitfalls, either as it is here proposed or with some modifications utilising some of the freedom still available. We shall find it interesting then to note that it has also some predictions which are probably accessible to - experimental tests in the not too distant future. There are first the dual gauge bosons and dual coloured Higgses. Crude estimates from our trial calculation of the CKM matrix reported in Section 6 suggest that dual gauge bosons may have masses in the several Tev range, and if so may be accessible to LHC. As for the masses of the dual coloured Higgses, however, we have at present no idea of their magnitudes. Secondly, there is the exciting possibility suggested at the end of Section 5 that there may be yet a deeper level of confinement than colour with dual weak isospin. If so, future deep-inelastic experiment at ultra-high energy may reveal internal structures to what are presently regarded as elementary objects such as quarks and leptons.

Finally, we remark that dual symmetry is claimed to be inherent to YangMills theory as it is to electromagnetism. If this is true, then its effects would be unavoidable, and even if one does not choose to interpret the internal symmetry frames as Higgs fields and dual colour as generation as we do here, the existence in theory of these niches as consequences of dual symmetry on the one hand, and the empirical requirement of Higgs fields and fermion generations on the other, would still have to be accounted for in some manner.

\section{Acknowledgement}

We are grateful to Bill Scott for teaching us some rudiments of the CKM matrix and to Dick Roberts, Ben Allanach, and Herbi Dreiner for helpful conversations on practicalities in running the fermion mass matrix. One of us (TST) thanks the Wingate Foundation for partial support.

\section{References}

[1] P A M Dirac, Proc. Roy. Soc. London A133, 60, (1931); Phys. Rev. 74, 817 (1948). 
[2] Julian Schwinger, Phys. Rev. 144, 1087, (1966); Phys. Rev. D12, 3105, (1975).

[3] D. Zwanziger, Phys. Rev. 176 1480, (1968); Phys. Rev. D6, 458, (1972).

[4] Tai-Tsun Wu and Chen-Ning Yang, Phys. Rev. D12, 3845, (1975); Phys. Rev. D14, 437, (1976).

[5] C. Montonen and D. Olive, Phys. Lett. 72B, 117, (1977).

[6] S. Deser, J. Phys. A15, 1053, (1982).

[7] E. Lubkin, Ann. Phys. (New York) 23, 233, (1963).

[8] S. Coleman, in New Phenomena in Subnuclear Physics, ed. by A. Zichichi, (Plenum, New York, 1976), p. 297.

[9] M. Halpern, Nucl. Phys. B139, 477, (1978).

[10] G. 't Hooft, Acta Physica Austrica suppl. XXII, 531, (1980).

[11] Chan Hong-Mo, P. Scharbach, and Tsou Sheung Tsun, Ann. Phys. (New York) 167, 454, (1986).

[12] N. Seiberg and E. Witten, Nucl. Phys. B426, 19 (1994); B431, 484 (1994).

[13] K. Intriligator and N. Seiberg, Nucl. Phys. B431, 551 (1994); N. Seiberg, ibid. B435, 129 (1995); N. Seiberg, in Particles, Strings, and Cosmology, Proceedings of the Workshop, Syracuse, New York, 1994, edited by K.C. Wali (World Scientific, Singapore, 1995).

[14] C. Vafa and E. Witten, Nucl. Phys. B431, 3 (1994); E. Witten, Math. Res. Lett. 1, 769 (1994).

[15] O. Aharony, Phys. Lett. 351B, 220 (1995); L. Girardello, A. Giveon, M. Porrati, and A. Zaffaroni, Nucl. Phys. B448, 127 (1995); A. Ceresole, R. D'Auria, S. Ferrara and A. Van Proeyen, Nucl. Phys. B444 92 (1995); 
J.A. Harvey, G. Moore and A. Strominger, Phys. Rev D52 7161, (1995); R.G. Leigh and M.J. Strassler, Nucl. Phys. B447, 95 (1995); E. Comay Nuovo Cim. B110, 1347, (1996); D. Kutasov and A. Schwimmer, Phys. Lett. 354B, 315 (1995); K. Intriligator and P. Pouliot, Phys. Lett. 353B, 471 (1995); K. Intriligator, Nucl. Phys. B448, 187 (1995); C. Gomez and E. Lopez, Phys. Lett. 357B, 558 (1995); E. Witten, hepth/9505186; G.W. Gibbons and D.A. Rasheed, Nucl. Phys. B454, 185 (1995); K. Intriligator, R.G. Leigh and M.J. Strassler, Nucl. Phys. B456, 567 (1995); E. Alvarez, L. Alvarez-Gaumé and I. Bakas, Nucl. Phys. B457, 3 (1995); Y. Lozano, Phys. Lett. B364, 19 (1995); C. Ford and I. Sachs, Phys. Lett. B362, 88 (1995); O. Aharony and S. Yankielowicz, Nucl. Phys. B473, 93, (1996); Kimyeong Lee, E.J. Weinberg and Piljin Yi, Phys. Lett. B376, 97, (1996); S.F. Hewson and M.J. Perry, hepth/9603015. P.J. Hodges and N. Mohammedi, hep-th/9608023; F. Ferrari, hep-th/9611012; J.H. Brodie and M.J. Strassler, hep-th/9611197. The above is still only a partial list of the many articles which have recently appeared on the subject.

[16] Chan Hong-Mo, J. Faridani and Tsou Sheung Tsun, Phys. Rev. D51, 7040, (1995); Phys. Rev. D53, 7293, (1996).

[17] Chan Hong-Mo, J. Faridani, and Tsou Sheung Tsun, Phys. Rev. D12, $7293,(1996)$.

[18] Chan Hong-Mo and Tsou Sheung Tsun, in preparation.

[19] For a review, see e.g. Chan Hong-Mo and Tsou Sheung Tsun, Some Elementary Gauge Theory Concepts (World Scientific, Singapore, 1993).

[20] Chen Ning Yang, Phys. Rev. D1, 2360, (1979).

[21] Chan Hong-Mo and Tsou Sheung Tsun, Nucl. Phys. B189, 364, (1981).

[22] See e.g. F.W. Heyl et al., Rev. Mod. Phys. 48, 393, (1976).

[23] See e.g. Review of Particle Physics ed. R.M. Barnett et al. Phys. Rev. D54, 1, (1996) as summarized in the data booklet. 
[24] Steven Weinberg, Phys. Rev. D7, 2887, (1973).

[25] See e.g. B. Grzadkowski, M. Lindner and S. Theisen, Phys. Lett. B198, 64, (1987). 